Schmerz 2016 $\cdot 30: 286-288$

DOI 10.1007/s00482-016-0111-z

Online publiziert: 11. April 2016

(c) The Author(s) 2016. This article is available

at SpringerLink with Open Access

CrossMark

\author{
L. Granata ${ }^{1,2} \cdot$ H. Niebergall' $\cdot$ R. Langner ${ }^{2} \cdot$ R. Agosti ${ }^{2} \cdot$ L. Sakellaris $^{3}$ \\ 'Schmerzmedizin Zürich, Zollikerberg, Schweiz \\ ${ }^{2}$ Kopfwehzentrum Hirslanden, Zürich, Schweiz \\ ${ }^{3}$ Augenchirurgie am Bahnhof, St. Gallen, Schweiz
}

\title{
Ketamin i. v. zur Behandlung von Clusterkopfschmerz
} Eine Beobachtungsstudie

Clusterkopfschmerzen haben eine Inzidenz von 1-3 pro 10.000 bei einem Geschlechterverhältnis männlich zu weiblich von 2,5:1. Die Erkrankung ist nicht lebensbedrohlich, aber die Attacken können den einzelnen Patienten erheblich beeinträchtigen und die Lebensqualität stark einschränken. Die Attacken dauern zwischen 15 min und $3 \mathrm{~h}$.

\section{》) Die Pathophysiologie von Clusterkopfschmerz ist unklar}

Die Pathophysiologie der Erkrankung ist unklar. Bekannt ist, dass der Hypothalamus und der Hirnstamm sowie genetische Faktoren wie der G1246A-Polymorphismus eine Rolle spielen. Oft charakterisiert ein zirkadianer Rhythmus die Attacken. Unterschieden wird zwischen episodischem und chronischem Clusterkopfschmerz. Die Standardtherapie erfolgt mit Kortison (kurzzeitig), Verapamil und bei mangelndem Ansprechen alternativ mit Valproat, Topiramat und Lithium. Die Attackentherapie erfolgt mit Sauerstoff und Triptane.

\section{Methodik}

Wir untersuchten in unserem Zentrum insgesamt 29 Patienten mit Clusterkopfschmerz, 2 Frauen und 27 Männer. Das Durchschnittsalter der Probanden lag bei 44 Jahren, wobei die Dauer der Erkrankung 1-18 Jahre betrug, verbunden mit diversen Arztkonsultationen. Die Patienten waren therapieresistent für herkömmliche Behandlungsansätze und Pharmaka, einige von ihnen auch für eine perikraniale Therapie mit Botulinumtoxin, die „off label“ eingesetzt wird.

In der Studiengruppe befanden sich 13 Patienten mit chronischem und $16 \mathrm{~Pa}$ tienten mit episodischem Clusterkopfschmerz. Bis auf 6 Patienten, die eine prophylaktische Basismedikation ablehnten, waren die Patienten auf bis dato weniger effektive Basistherapien eingestellt, z. B. auf den Kalziumkanalblocker Verapamil (240-480 mg/Tag), das Phasenprophylaktikum Lithium (Blutspiegel 0,5-1,2 mmol/l), den Natriumkanalblocker Valproat (1400 mg/Tag) und das Antikonvulsivum Topiramat (bis zu $200 \mathrm{mg} / \mathrm{Tag}$ ).

Einige der Patienten verblieben bei konstanten Dosierungen von Kortisonpräparaten, z. B. Prednisolon. Der Großteil der Patienten nutzte in der Akutsituation bei 2-8 Attacken/Tag Triptane, mit oder ohne Sauerstoffgabe über Reservoirmaske, ein Patient, der auf Triptane nicht angesprochen hatte, nahm Morphinpräparate und ein Patient Novaminsulfon er hatte damit Erfolg und wollte die Therapie bei der Erstvisite nicht ändern.

Die Attackenfrequenz varierte in beiden Gruppen zwischen 2 und 8 pro Tag. Bei den chronischen Patienten betrug die Dauer der Kopfschmerzattacken 2-18 Jahre. Bei den episodischen Patienten lag die Dauer der Episode bei einigen Tagen bis zu 1-2 Monaten.

Alle Patienten dokumentierten die Kopfschmerzen mittels Kopfschmerztagebuch in Papierform oder digital. Eine routinemäßige Bestimmung von De- pressions- oder Lebensqualitätsscores wurde nicht durchgeführt. Die klinische Beurteilung zeigte allerdings, dass bei allen Patienten die Lebensqualität durch die Attacken deutlich eingeschränkt war. Suizidale Gedanken waren nicht selten.

Wir applizierten im Rahmen eines Krankenhaussettings i.v. eine geringe analgetische Dosis des N-Methyl-D-Aspartat-Rezeptor-Antagonisten Ketamin (Racematenantiomere S und R). Appliziert wurden $0,5 \mathrm{mg} / \mathrm{kg}$ über $40 \mathrm{~min}$ bis $\mathrm{zu} 1 \mathrm{~h}$, alle 2 Wochen, je nach Ansprechen erfolgten 1-4 Applikationen. Einige Patienten mit episodischer Verlaufsform des Clusterkopfschmerzes erhielten die zweite Applikation in kürzerem Abstand, d.h. 2-4 Tage später. Alle Patienten bekamen Ketamin außerhalb der Attacken. Die Uhrzeit der Verabreichung war unterschiedlich, meist erfolgte die Applikation morgens. Ein Patient entwickelte eine kurze Attacke zu Beginn der Infusion. Die Infusion wurde nicht unterbrochen.

Die Verabreichung von Ketamin i.v. wurde als Akuttherapie eingesetzt, um die Cluster der Attacken zu stoppen. Gleichzeitig erhofften wir uns aber auch eine prophylaktische Wirkung.

Etwa ein Drittel der Patienten erhielt Midazolam 2,5 mg i. v. als Prämedikation vor der Ketamingabe. Der Grund hierfür lag in der Reduktion möglicher Nebenwirkungen des Präparats, z. B. von Halluzinationen, Angstattacken oder Albträumen.

Ausschlusskriterien waren ein Alter $<18$ Jahren, Schwangerschaft, kognitive Defizite, ein unkontrollierter Hypertonus 
und jegliche Form von Blockbildern im Elektrokardiogramm.

\section{Ergebnisse}

Der Großteil der Patienten tolerierte die Infusion gut. Milde Symptome von Derealisation traten auf, die Sekunden bis wenige Minuten andauerten. Diese waren ein guter prognostischer Faktor. Strategien zur Beruhigung wurden entwickelt, z. B. das „Handhalten“ durch einen Angehörigen, eine Maßnahme, die im Allgemeinen innerhalb kürzester Zeit half. Nur bei einem Patienten mit stärkerer Dissoziation musste nachfolgend Midazolam gespritzt werden, woraufhin sich die Symptomatik verbesserte. Ein Patient entwickelte vorübergehend eine arterielle Hypertonie. Bei kurzzeitiger Unterbrechung der Infusion, gefolgt von einer langsameren Verabreichung, normalisierte sich der Blutdruck ohne medikamentöse Intervention.

Zwei Patienten entwickelten passagere bradykarde Phasen. Im einen Fall handelte es sich um die erste Infusion; diese musste beendet werden. Der Patient hatte kurz vor der Verabreichung zum sechsten Mal an diesem Tag Sumatriptan eingenommen, was eventuell eine Rolle spielte. Vor der zweiten und dritten Infusion wurde die Sumatriptaneinnahme nicht gestattet. Die Behandlung verlief dann ohne Probleme. Beim zweiten Patienten war die Bradykardie kurz, passager und nicht interventionsbedürftig.

Alle Patienten zeigten nach Ketaminapplikation mäßige bis starke Müdigkeit, die bis $\mathrm{zu} 24 \mathrm{~h}$ andauerte.

Durch die Infusion war es möglich, bei 7 von 13 Patienten (54\%) mit chronischem Clusterkopfschmerz die Attacken komplett zu beenden, entweder sofort oder 1-2 Wochen nach der letzten Infusion, wobei die Dauer der Attackenfreiheit zwischen 3 und 18 Monaten variierte, je nach Zeitpunkt der Verabreichung in der Studie - der vorliegende Beitrag wurde 18 Monate nach Studienbeginn erstellt. Ein Patient sprach teilweise an, d.h., Frequenz und Intensität der Attacken nahmen nur ab. Vier Patienten zeigten keinerlei Verbesserung, ein Patient beendete die Studie vorzeitig.

Schmerz 2016 · 30:286-288 DOI 10.1007/s00482-016-0111-z

(c) The Author(s) 2016

L. Granata · H. Niebergall · R. Langner $\cdot$ R. Agosti $\cdot$ L. Sakellaris

\section{Ketamin i. v. zur Behandlung von Clusterkopfschmerz. Eine Beobachtungsstudie}

\section{Zusammenfassung}

Clusterkopfschmerzen haben eine Inzidenz von 1-3 pro 10.000 bei einem Geschlechterverhältnis männlich zu weiblich von 2,5:1. Die Erkrankung ist nicht lebensbedrohlich, aber die Attacken können den einzelnen Patienten erheblich beeinträchtigen und die Lebensqualität stark einschränken. Die Attacken dauern zwischen 15 min und $3 \mathrm{~h}$. Die Pathophysiologie der Erkrankung ist unklar. Bekannt ist, dass der Hypothalamus und Hirnstamm sowie genetische Faktoren wie der G1246A-Polymorphismus eine Rolle spielen. Oft charakterisiert ein zirkadianer Rhythmus die Attacken. Unterschieden wird zwischen episodischem und chronischem Clusterkopfschmerz. In unserer Studie behandelten wir 29 Patienten mit Clusterkopfschmerz - 13 mit der chronischen und 16 mit der episodischen Form. Die Patienten erhielten Ketamin i.v. über 40 min bis eine Stunde alle 2 Wochen oder zeitnäher bis zu 4-mal. Es gelang, bei $100 \%$ der Patienten mit episodischen und bei $54 \%$ der Patienten mit chronischen Clusterkopfschmerzen die Attacken für einen Zeitraum von 3 bis 18 Monaten zu stoppen. Wir postulierten neuroplastische Phänomene und eine Neuromodulation als mögliche Mechanismen.

\section{Schlüsselwörter}

Ketamin · N-Methyl-D-Aspartat-Rezeptor Antagonist · Neuromodulation · Neuroplastizität . Lebensqualität

\section{Ketamine i. v. for the treatment of cluster headaches. An observational study}

\section{Abstract}

Cluster headaches have an incidence of 1-3 per 10,000 with a 2.5:1 male-to-female gender ratio. Although not life threatening, the impact of the attacks on the individual patient can result in tremendous pain and disability. The pathophysiology of the disease is unclear, but it is known that the hypothalamus, the brainstem, and genetic factors, such as the G1246A polymorphism, play a role. A distinction is made between episodic and chronic cluster headaches. In a controlled setting, we treated 29 patients with cluster headaches ( 13 with chronic cluster and 16 with the episodic form), who had been refractory to conventional treatments, with a low dose of ketamine (an NMDA receptor antagonist) i.v. over 40 min to one hour every 2 weeks or sooner for up to four times. It was observed that the attacks were completely aborted in $100 \%$ of patients with episodic headaches and in $54 \%$ of patients with chronic cluster headaches for a period of 3-18 months. We postulated neuroplastic brain repair and remodulation as possible mechanisms.

\section{Keywords}

Ketamine - N-Methyl-D-Aspartate Receptor Antagonist · Neuromodulation . Neuroplasticity · Quality of Life
Bei 13 Patienten mit episodischem Clusterkopfschmerz (100\%) konnte eine Beendigung der Episode erreicht werden, wobei ein Patient bereits unter der Standardmedikation - vor der Ketamininfusion - eine Reduktion der Symptomintensität und -frequenz verspürt hatte. Alle Patienten behaupteten, dass die Behandlung mit Ketamin die Episode deutlich verkürzte. Zum Vergleich wurden andere Episoden herangezogen, die beispielsweise 1-mal pro Jahr für 4 Monate auftraten.
Bereits in den Infusionsintervallen reduzierten sich die Frequenz und Intensität der Attacken. Als Ansprechen auf die Ketamininfusion betrachteten wir ein komplettes Sistieren der Attacken, sowohl bei chronischen als auch bei episodischen Clusterkopfschmerzen.

Die Nachbeobachtungszeit betrug bis zum Zeitpunkt der Manuskripterstellung 18 Monate. Bei 3 Patienten wurde 6 Monate nach der Erstinfusion eine BoosterBehandlung durchgeführt. Die Ketamindosis war die gleiche wie zuvor. 
Die besten Resultate ergaben sich bei Patienten mit episodischem Clusterkopfschmerz, die zeitnah zu Beginn der Episode behandelt wurden. Bei kürzeren Behandlungsintervallen und bei den bereits vorab mit perikranialem Botulinumtoxin behandelten Patienten waren die Ergebnisse ebenfalls besser, das Ansprechen war schneller und die Analgesie effektiver. Die Botulinumtoxinbehandlung hatte zwar die Attacken davor nicht zum Sistieren gebracht, die Patienten aber „empfänglicher" für Ketamin gemacht.

Zwei der Patienten waren Nonresponder und hatten vorab keine Basismedikation eingenommen. Die Triptaneinnahmefrequenz im Vorfeld bei den Patienten mit chronischem Clusterkopfschmerz scheint ebenso einen Einfluss zu haben: Je höher die Gesamtdosis von Sumatriptan war, desto geringer war die Symptomverbesserung.

Der Mechanismus der Ketaminwirkung beinhaltet eine Neuroregeneration, eine Remodulation, Interaktionen mit biogenen Aminen sowie die Entkoppelung des thalamokortikalen vom limbischen System. Zusätzlich kann dadurch die Wiederaufnahme von Katecholaminen und Serotonin gehemmt werden. Es bestehen Interaktionen mit muskarinergen, nikotinergen und $\gamma$-Aminobuttersäure(GABA)-Rezeptoren. Weiterhin kann die Produktion von Stickstoffmonoxid und zyklischem Guanosinmonophosphat (cGMP) über alpha-amino3-hydroxy-5-methyl-4-isoxazolepropionic acid (AMPA)-Rezeptoren verringert werden. Ob ein Effekt am Opioidrezeptor besteht, ist noch nicht vollständig geklärt [1-3].

\section{Diskussion}

Die vielversprechenden Ergebnisse zeigen, dass die Lebensqualität der oben beschriebenen Patienten deutlich verbessert werden konnte. Die Wirkungsdauer hielt zwischen 6 und 18 Monaten an.

Es gibt kaum Literatur zur Gabe von Ketamin bei Kopfschmerz, nur Einzelfälle wurden beschrieben. Die Durchführung weiterer Studien beim Clusterkopfschmerz mit einer höheren Patientenzahl und mit Placebokontrollen wäre sinnvoll.
In einer kleinen Gruppe von Patienten mit Migräne und chronischen täglichen Kopfschmerzen („Chronic Daily Headaches"), die wir ebenso mit Ketamin i.v. behandelten, waren die Ergebnisse bisher weniger überzeugend. Diese Patienten hatten vorab bereits auf diverse konventionelle Behandlungsansätze nicht ausreichend angesprochen.

Andererseits findet man in der Literatur Informationen über den Effekt von Ketamin bei der Behandlung von therapierefraktären Depressionen. Unsere bisherigen Erfahrungen bestätigen den positiven Effekt auf die depressive Symptomatik.

\section{Korrespondenzadresse}

\section{Dr. L. Granata}

Schmerzmedizin Zürich

Trichtenhauser Str. 12, 8125 Zollikerberg, Schweiz

livia.granata1961@gmail.com

\section{Einhaltung ethischer Richtlinien}

Interessenkonflikt. L. Granata, H. Niebergall, R. Agosti, R. Langner und L. Sakellaris geben an, dass kein Interessenkonflikt besteht.

Alle beschriebenen Untersuchungen am Menschen wurden mit Zustimmung der zuständigen Ethik-Kommission, im Einklang mit nationalem Recht sowie gemäß der Deklaration von Helsinki von 1975 (in der aktuellen, überarbeiteten Fassung) durchgeführt. Von allen beteiligten Patienten liegt eine Einverständniserklärung vor.

Open Access. This article is distributed under the terms of the Creative Commons Attribution 4.0 International License (http://creativecommons.org/ licenses/by/4.0/), which permits unrestricted use, distribution, and reproduction in any medium, provided you give appropriate credit to the original author(s) and the source, provide a link to the Creative Commons license, and indicate if changes were made.

\section{Literatur}

1. Li N, Lee B, Liu RJ, Basnasr M, Dwyer JM, Iwata M et al (2010) mTORdependent synapse formation underlies the rapid antidepressant effects ofNMDA antagonists. Science (80-) 329:959-964

2. Monteggia LM, Zarate C Jr. (2015) Antidepressant actions of ketamine: from molecular mechanisms to clinical practice. Curr Opin Neurobiol 30:139-143

3. Kristal JH, Sanacora G, Duman RS (2013) Rapid-acting glutamatergic antidepressants:the path to ketamine and beyond. Biol Psychiatry 73(12):1133-1141

\section{Palliativmedizin in der Pneumologie}

Zur Verbesserung der Lebensqualität von Patienten und ihren Angehörigen, umfasst die Palliativmedizin im wesentlichen Symp-

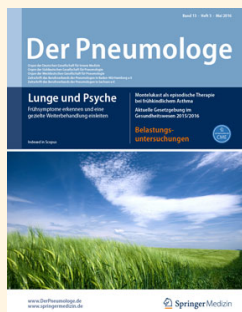
tomkontrolle, psychosoziale Unterstützung und Versorgungsplanung. Bei Atemnot bestehen zwar neurophysiologisch Ähnlichkeiten mit

der Schmerzempfindung, aber sie ist erheblich komplexer als Schmerz und in vielen Bereichen schwieriger zu verstehen. In Der Pneumologe 02/2016 finden Sie Vorschläge für eine bessere palliativmedizinische Versorgung von Patienten mit chronischen, nicht heilbaren pulmonalen Erkrankungen, sowie auch für die Integration der Palliativmedizin in ein Lungentransplantationsprogramm, auch wenn Transplantations- und Palliativmedizin ähnlich wie die Intensivmedizin auf den ersten Blick gegensätzliche Ansätze haben.

- Palliativmedizin in der thorakalen Onkologie

- Palliativmedizin bei nicht malignen chronisch pulmonalen Erkrankungen

- Ethik und Palliativmedizin in der Intensiv- und Beatmungsmedizin

- Palliative Care in der Lungentransplantation

Bestellen Sie diese Ausgabe zum Preis von 39,-EUR zzgl. Versandkosten bei Springer Customer Service Center Tel.: +49 6221-345-4303

E-Mail: leserservice@springer.com

Suchen Sie noch mehr zum Thema? Mit e.Med, dem Kombi-Abo von Springer Medizin, können Sie schnell und komfortabel in über 600 medizinischen Fachzeitschriften recherchieren.

Weitere Infos unter www.springermedizin.de/eMed 